\title{
Correlations between subject generated letter frequencies and observed frequencies in English'
}

\author{
M. S. Mayzner, M. E. Tresselt, S. Adler and K. M.' Sehoenberg \\ NEW YORK UNIVERSITY
}

\begin{abstract}
Five groups of $10 \mathrm{Ss}$ each were asked to randomly generate 1000 single letters, 500 digrams, 334 trigrams, 250 tetragrams, and 200 pentagrams. Correlations between generated single letter frequencies and observed frequencies in English were high, ranging from .70 to .83 , and it was concluded that Ss do not operate as random letter generators, but rather reflect quite precisely single letter frequencies as found in the language.

\section{Problem}

Some years ago Attneave (1953) examined the degree to which Ss can judge the frequency with which single letters occur in the language by asking Ss to make numerical judgments of the relative frequence with which each letter of the alphabet occurs in English. He found a correlation of .79 between median .judged frequency and English-text frequency, based on a sample size of 10,341 letters. Attneave also found appreciable correlations, where the task involved guessing the identities of 100 fictitious letters in a pseudo-ESP experiment. For a different task, in which Ss attempted to form words by guessing letters one at a time until the word was completed, Battig (1957) found a definite tendency for the frequency of guessed letters to correspond to the frequency of occurrence of these letters in English. In still other tasks, involving judgments of the frequency of single letters, digrams, and trigrams, and letter association techniques (Mayzner \& Tresselt, 1962a; Underwood \& Schulz, 1960), additional evidence has been found strongly suggesting that Ss are very sensitive to the relative frequencies with which single letters, as well as digrams and trigrams occur in English.

The present study is intended to obtain further evidence bearing on the above issue. The assumption is made that as a result of an individual's past history of experience with his natural language a language response hierarchy develops which reflects quite precisely the frequencies of letter sequences in the language, and in particular single letter frequencies. Such an assumption suggests that if we treat $S$ as a random letter generator and ask him to generate a large sample of letters randomly, he will, in fact, not be able to do so (i.e., he will behave non-randomly) and will produce letters in correspondence with their frequency of occurrence in the language.

\section{Method}

Five different groups (A, B, C, D, and E) of $10 \mathrm{Ss}$ each were employed. Group A was instructed to write down as quickly as possible 1000 letters, being sure to use all letters of the alphabet and to do so in a completely random fashion. Instructions to Groups B, C, D, and $\mathrm{E}$ were identical, except Group B was told to generate 500 random digrams, Group $C$ was told to generate 334 random trigrams, Group D was told to generate 250 random tetragrams, and Group $\mathrm{E}$ was told to generate 200 random pentagrams. Ruled recording sheets were employed consisting of $1000,500,334,250$, and 200 cells, respectively, for Groups A, B, C, D, and E.

\section{Results and Discussion}

Since each S produced 1000 letters, the total number of As, Bs, Cs, etc., were summed for the $10 \mathrm{Ss}$ in each group, and Rhos were calculated between the frequency values for the generated letters and the frequency values for single letters as obtained from the Mayzner and Tresselt tables (1962b) based on 20,000 words or 87,250 single letters. The Rhos for Groups A, B, C, D, and E were $.76, .75, .83, .80$, and .70 , respectively (Rho, .69; $\mathrm{p}<.001)$.

Since the Mayzner and Tresselt tables also give single letter frequency values tabulated separately for wordlength and letter-position variations, Rhos were calcu-

Table 1. Rank-Difference Correlations (Rhos) between Subject Generated and Single-Letter Frequency Table Values

Sub-Total Word Frequency

Letter Position Frequency

\begin{tabular}{|c|c|c|c|c|c|c|}
\hline & & 1 & $\underline{2}$ & 3 & 4 & 5 \\
\hline Trigrams & $.74(.94)$ & $.56(.62)$ &. $.77(.82)$ & $.60(.61)$ & & \\
\hline Tetragrams & $.77(.95)$ & $.69(.54)$ & $.76(.72)$ & $.82(.74)$ & $.70(.70)$ & \\
\hline Pentagrams & $.72(.97)$ & $.58(.49)$ & $.63(.80)$ & $.66(.76)$ & $.65(.81)$ & $.48(.66)$ \\
\hline
\end{tabular}


lated separately, between generated and table values, for Groups C, D, and E, i.e., those groups producing trigrams, tetragrams, and pentagrams, and these values are presented in Table 1. Also, presented in Table 1 in parentheses are the Rhos obtained between the sub-total (i.e., sub-total frequencies for three, four, and five letter words) as well as the letter position frequency table values and grand total table values (i.e., the values based on the total sample size of 87,250 letters). In other words, the Rhos in parentheses are correlations internal to the Mayzner and Tresselt tables and indicate the degree to which grand total letter frequencies correlate with letter frequency sub-totals in three, four, and five letter words (shown under the Sub-Total Word Frequency column) and in particular positions in such words (shown under the Letter Position Frequency columns).

The overall results suggest very strongly that Ss do not operate or behave as random letter generators, but rather reflect quite precisely the frequencies with which single letters occur in the language, and are in agreement with earlier findings (Attneave, 1953; Battig, 1957; Mayzner \& Tresselt, 1962a; Underwood \& Schulz, 1960). Further, having Ss generate digrams, trigrams, etc., does not alter the basic frequencies associated with single letter generation, as shown by the approximately equal Rho values found for Groups A, B, C, D, and E. Finally, the Rhos in parentheses given in Table 1 show that for letter positions beginning and ending three, four, or five letter words, there is a drop in correlation values compared to letter positions within the word. Since this pattern also is reflected in the Rhos for generated letters, it would appear that instructing Ss to generate trigrams, tetragrams, or pentagrams does not change their basic letter generation model to reflect these finer positional variations, but rather they continue generating letters in accordance with the overall frequencies with which single letters occur in the total sample. However, in an earlier study (Mayzner \& Tresselt, 1962a) evidence was found indicating Ss are highly sensitive to word-length and letter-position variations, which suggests that random letter generation may not be as sensitive a technique as the previously used judgmental task in assessing letter sequence response hierarachies.

\section{Referenees}

ATTNEAVE, F. Psychological probability as a function of experienced frequency. J.exp. Psychol., 1953, 46, 81-86.

BATTIG, W. F. Some factors affecting performance on a word-formation problem. J.exp. Psychol., 1957, 54, 96-104.

MAYZNER, M. S., \& TRESSELT, M. E. The ranking of letter pairs and single letters to match digram and single-letter frequency counts. J.verb. Learn. verb. Beh., 1962(a), 1, 203-207.

MAYZZNER, M. S., \& TRESSELT, M. E. Tables of digram and single-letter frequency counts by word-length and letter-position for a sample of 20,000 words. Mimeographed copy, 1962(b), New York University Library.

UNDERWOOD, B. J., \& SCHULZ, R. W. M e a ning fulness and verbal learning. Philadelphia: Lipincott, 1960 .

\section{Note}

1. This research was supported in part by Contract Nonr 285(56) between the Engineering Psychology Branch of the Office of Naval Research and New York University. 